\title{
Interacting laser and Bose-Einstein-condensate atomic beams: Mutual guiding structures
}

\author{
F. Cattani, ${ }^{1, *}$ V. Geyko, ${ }^{2}$ A. Kim, ${ }^{2}$ D. Anderson, ${ }^{3}$ and M. Lisak ${ }^{3}$ \\ ${ }^{1}$ Department of Physics, Clarendon Laboratory, OX1 3PU, Oxford, United Kingdom \\ ${ }^{2}$ Institute of Applied Physics, Russian Academy of Sciences, 603950 Nizhny Novgorod, Russia \\ ${ }^{3}$ Department of Radio and Space Science, Chalmers University of Technology, SE-412 96 Göteborg, Sweden
}

(Received 29 October 2009; published 22 April 2010)

\begin{abstract}
A basic set of equations describing the interaction of a Bose-Einstein condensate (BEC) with a laser field is derived based on a semiclassical model and applied to the problem of mutual guiding of laser and BEC atomic beams. Within this framework we have studied stationary spatially localized solutions of the nonlinear system which describe possible laser and BEC atomic beam guiding and have shown their stability as well. It is also shown that a self-guiding effect can be realized through both single- and multiple-scaled structures of a BEC atomic and a laser beam.
\end{abstract}

DOI: 10.1103/PhysRevA.81.043623

PACS number(s): $03.75 . \mathrm{Nt}$, 42.50.Ct

\section{INTRODUCTION}

From the points of view of both applications and theoretical modeling, the behavior of Bose-Einstein condensates (BECs) in laser fields has brought new challenges in the field of atom optics. The physics of the atom-laser interplay involves two aspects of the same phenomenon: On the one hand, there is the effect of the electromagnetic radiation on atoms showing long-range coherence and significant collective behavior. On the other hand, there is the effect of the atoms on the same radiation. Depending on which aspect is emphasized, different physical scenarios can be investigated, ranging from detection mechanisms for BECs based on the way the atoms affect propagating light [1] to the creation of spatially localized atomic structures exploiting the action of laser light on a BEC [2].

Several authors have worked on this problem (see, for instance, [3]), and by deriving a description of a two-level atom interacting with monochromatic electromagnetic radiation, they have shown what kind of mathematical structures can be expected. The same problem was analyzed within a semiclassical framework in the spirit of mean-field theory [4], leading to a system of equations for the coupled dynamics of atoms and laser light. The numerical analysis presented in [4], under the assumption of the slowly varying envelope approximation, has shown the tendency of this system to settle down onto stationary states with mutual atom-laser localization for the spatial structures. This is an interesting effect from the point of view of atom manipulation by light, and therefore it seems important to investigate the existence of purely stationary, localized solutions for the system. This is the aim of the present investigation.

The paper is organized as follows. In Sec. II we introduce the model used in the present investigation to describe the interaction of BECs with laser fields, including the vectorial character of the field. We then discuss the approximations considered in the analysis of the mutual guiding phenomena of laser and BEC atomic beams and then formulate the stationary form of the model equations. These equations are important since they describe all possible stationary solutions, for example, spatially localized structures or structures in optical lattices. In Sec. III, we focus on the stationary regime and consider in particular the effects of mutual atom-laser guiding and the concomitantly generated structures. Some aspects of the stability problem are analyzed in Sec. IV.

\section{BASIC EQUATIONS}

The interaction of electromagnetic radiation and a coherent matter field has been described from first principles within the framework of quantum field theory (see Krutitsky et al. [3]). What we would like to present here is the derivation of a model for the same interaction based on semiclassical reasoning. This kind of reasoning can be seen as complementary to a fully quantum model for the atoms and may help in gaining insight into the physical processes going on during the interaction. It is important to notice that the two derivations lead to equations for the atoms that are in complete agreement, once the natural limitations of the semiclassical model, which will be discussed in the following, are taken into account.

The basic idea behind a semiclassical model to describe the atom-laser interaction consists in modeling the force exerted by laser light on an atom and the refractive index experienced by the laser due to the presence of the atoms [5]. For the force, Helmholtz's calculation of the work done by an electric field on a dielectric [6] has been generalized by Pitaevskii [7] to a quasimonochromatic field $\mathcal{E}(\mathbf{r}, t)=\operatorname{Re}[\mathbf{E}(\mathbf{r}, t) \exp (-i \omega t)]$, where $\mathbf{E}(\mathbf{r}, t)$ is the complex amplitude of the laser field considered as slowly varying in time. It is thus found that the time-averaged force (over laser cycles) is

$$
\mathbf{F}=\frac{1}{16 \pi} \nabla\left(|\mathbf{E}|^{2} \frac{\partial \epsilon}{\partial n}\right)=-\nabla V_{d},
$$

where $\epsilon(\omega, n)$ is the medium dielectric constant at atom density $n$; that is, in this case it describes the nature of the atomic medium. This is the point in the derivation where the quantum nature of the system enters, and the dielectric constant is given by

$$
\epsilon(\omega, n)=1+\frac{4 \pi \alpha n}{1-\frac{4 \pi}{3} \alpha n},
$$

\footnotetext{
*f.cattani1@physics.ox.ac.uk
} 
where, as derived from quantum theory, $\alpha(\omega)=-d^{2} / \hbar \Delta$ is the atomic polarizability at the laser frequency $\omega$, with $\Delta=\omega-\omega_{a}$ being the detuning from the nearest atomic resonance frequency $\omega_{a}$, and $d$ is the dipole matrix element of the resonant transition (for the quantum derivation, see [3], and for the classical one, see [8]). By accepting this model we also accept its limitations. In particular, since the concept of force is a purely classical one, quantum fluctuations, stochastic heating, and any incoherent processes are to be neglected and the model can describe stationary structures only for large detunings $|\Delta| \gg \omega_{a}, \Gamma$ (where $\Gamma$ is the atom's natural linewidth). Use of the expression for the dielectric constant given in Eq. (2) in the macroscopic Maxwell equations for the electromagnetic field corresponds to the classical macroscopic electrodynamics limit of large atom numbers [8,9], which implies a difference between the local field (the microscopic field acting on an atom) and the macroscopic field (the field averaged over a volume containing many atoms). Under the limitations already mentioned, the potential $V_{d}$ that can be derived from Eq. (1) together with Eq. (2) can be inserted into the atomic Gross-Pitaevskii equation. There it describes the laser-induced dipole-dipole interaction between the atoms, that is, the fact that the incident radiation brings about absorption and emission of photons, which are then absorbed by other atoms, thus giving rise to a long-range interaction and acting as the analog of a nonlinear medium for the atomic wave function:

$i \hbar \frac{\partial \Psi}{\partial t}=\hat{H}_{0} \Psi+\left(U_{0}|\Psi|^{2}-\frac{\alpha}{4} \frac{|\mathbf{E}|^{2}}{\left(1-\frac{4 \pi}{3} \alpha|\Psi|^{2}\right)^{2}}\right) \Psi$.

Here $\hat{H}_{0}$ is the linear single-particle Schrödinger Hamiltonian, the wave function $\Psi$ is normalized as $N=\int|\Psi|^{2} d \mathbf{r}$ with $N$ denoting the total number of atoms, so that the gas density is $n=|\Psi|^{2}, U_{0}=4 \pi \hbar^{2} a_{s} / m, m$ is the atom mass, and $a_{s}$ is the $s$-wave scattering length, which can be either positive or negative (repulsive or attractive collisional interaction). Again, we would like to stress the fact that this equation, once the limits of our semiclassical derivation are taken into account, is the same equation derived from a fully quantum model in Ref. [3].

For the laser dynamics, its governing equation is the macroscopic wave equation

$$
\nabla \times \nabla \times \mathbf{E}+\frac{\omega^{2} \epsilon}{c^{2}} \mathbf{E}+\frac{i}{c^{2}} \frac{\partial\left(\omega^{2} \epsilon\right)}{\partial \omega} \frac{\partial \mathbf{E}}{\partial t}=\mathbf{0} .
$$

Thus Eqs. (2)-(4) constitute a basic self-consistent set of equations describing mutual laser-BEC-atomic-beam dynamics. The effects of coherent dynamics are consistently accounted for by this model, which describes the interaction in a very general way, from electrodynamical effects on BECs in optical lattices to the possibility of atom manipulation and guiding by light.

\section{A. Stationary regime}

The importance of the model from the point of view of realizable structures is related to the existence of stationary solutions and to their stability. In order to study the stationary regime of the interaction, we assume

$$
\begin{gathered}
\Psi(\mathbf{r}, t)=\Phi(\mathbf{r}) \exp \left(-i \frac{E_{a}}{\hbar} t\right), \\
\mathbf{E}(\mathbf{r}, t)=\mathbf{A}(\mathbf{r}),
\end{gathered}
$$

where $E_{a}$ plays the role of the chemical potential. Together with Eq. (2) and with use of $\nabla \cdot(\varepsilon \mathbf{E})=0$, the set of governing equations becomes

$$
\begin{gathered}
\hat{H}_{0} \Phi+\left(U_{0}|\Psi|^{2}-\frac{\alpha}{4} \frac{|\mathbf{A}|^{2}}{\left(1-\frac{4 \pi}{3} \alpha|\Phi|^{2}\right)^{2}}\right) \Phi=E_{a} \Phi, \\
\nabla^{2} \mathbf{A}+\left(\frac{\nabla \epsilon}{\epsilon} \cdot \mathbf{A}\right)+k^{2}\left(1+\frac{4 \pi \alpha|\Phi|^{2}}{1-\frac{4 \pi}{3} \alpha|\Phi|^{2}}\right) \mathbf{A}=\mathbf{0},
\end{gathered}
$$

which provides a general description of all possible stationary configurations of a BEC interacting with a laser field. Here $k=\omega / c=\frac{2 \pi}{\lambda_{L}}$ is the laser wave number in vacuum, $\lambda_{L}$ is the laser wavelength, and $E_{a}$ is the single-particle energy.

\section{SELF-GUIDING STRUCTURES}

\section{A. The model}

Of particular interest are the possible scenarios of mutual guiding of laser and BEC atomic beams. To investigate the self-guiding effects of mutual propagation, we consider a BEC atomic beam in free space copropagating with a laser beam. Under the realistic assumption that $L_{n} \gg \lambda_{L}$ (where $L_{n}$ is the characteristic length scale of transverse density modulations), we can consider a scalar model since $\boldsymbol{\nabla} \cdot \mathbf{E} \simeq 0$, as inferred from $\nabla \cdot(\epsilon \mathbf{E})=0$ or $\frac{\nabla \epsilon}{\epsilon} \cdot \mathbf{E} \simeq 0$. The subsequent class of solutions corresponds to mutual guiding in the form of long-distance localized beam propagation. To analyze it, we focus on modes localized in the transverse direction, the analogs of the well-known Kerr spatial solitons:

$$
\begin{aligned}
& \mathbf{A}(\mathbf{r})=a\left(\mathbf{r}_{\perp}\right) \exp (i h z) \mathbf{e}, \\
& \Phi(\mathbf{r})=\phi\left(\mathbf{r}_{\perp}\right) \exp \left(i h_{\phi} z\right),
\end{aligned}
$$

where $\mathbf{r}_{\perp}$ denotes the dimensions transverse to the propagation direction $z, \mathbf{e}$ is the polarization vector of the field, and $h$ and $h_{\phi}$ are the laser and atom propagation constants, respectively. These solutions must satisfy the fully stationary equations derived from (7) and (8), which written in normalized variables become

$$
\begin{gathered}
\tilde{\nabla}_{\perp}^{2} \tilde{\phi}-\beta \tilde{\phi}^{3}+\frac{s \tilde{a}^{2} \tilde{\phi}}{\left(1-s \tilde{\phi}^{2}\right)^{2}}-2 \mu^{2} \kappa_{\phi} \tilde{\phi}=0 \\
\tilde{\nabla}_{\perp}^{2} \tilde{a}+\frac{3 s \tilde{\phi}^{2} \tilde{a}}{1-s \tilde{\phi}^{2}}-2 \kappa \tilde{a}=0 .
\end{gathered}
$$

The normalization used is $\tilde{\mathbf{r}}=\mathbf{r} k$; for the atom wave function $\tilde{\phi}=\phi / \phi_{*}$ with $(4 \pi|\alpha| / 3) \phi_{*}^{2}=1$, and for the laser $\tilde{a}=a / a_{*}$ with $m|\alpha| a_{*}^{2} /\left(2 \hbar^{2} k^{2}\right)=1, s=\operatorname{sgn}(\alpha)$, and $\beta=$ $6 a_{s} /\left(k^{2}|\alpha|\right)$. The tilde will be dropped hereafter unless otherwise stated. Furthermore, $\mu=k_{a} / k, k_{a}=\sqrt{2 m E_{a}} / \hbar$, $\kappa_{\phi}=\left(h_{\phi}^{2} / k_{a}^{2}-1\right) / 2$, and $\kappa=\left(h^{2} / k^{2}-1\right) / 2$. Both $a(\mathbf{r})$ and $\phi(\mathbf{r})$ are real amplitudes and, for simplicity, we will consider 
only one transverse dimension $\mathbf{r}_{\perp}=x$. In addition, in what follows we will consider $\mu=1$ and $\beta \simeq 38$, corresponding, for instance, to a detuning of $100 \Gamma$ for ${ }^{87} \mathrm{Rb}$ atoms.

Equations (11) and (12) have a first integral, obtained by multiplying them, respectively, by $d \phi / d x$ and $d a / d x$ and then integrating the two equations over $x$ as

$$
\begin{gathered}
\frac{1}{2}\left(\frac{d \phi}{d x}\right)^{2}-\mu^{2} \kappa_{\phi} \phi^{2}-\frac{\beta}{4} \phi^{4}+\int \frac{s a^{2} \phi(d \phi / d x)}{\left(1-s \phi^{2}\right)^{2}} d x=c_{\phi}, \\
\frac{1}{2}\left(\frac{d a}{d x}\right)^{2}-\kappa a^{2}+\int \frac{3 s \phi^{2} a(d a / d x)}{1-s \phi^{2}} d x=c_{\chi}
\end{gathered}
$$

where $c_{\phi}$ and $c_{\chi}$ are two integration constants. The primitives of the two integrals left are connected by

$$
\int \frac{s a^{2} \phi(d \phi / d x)}{\left(1-s \phi^{2}\right)^{2}} d x=\frac{s}{2} \frac{a^{2} \phi^{2}}{1-s \phi^{2}}-\int \frac{s \phi^{2} a(d a / d x)}{1-s \phi^{2}} d x .
$$

The very last integral can be calculated from (14) and this leads to

$$
\begin{gathered}
\frac{1}{2}\left(\frac{d \phi}{d x}\right)^{2}+\frac{1}{2}\left(\frac{d \chi}{d x}\right)^{2}+\Pi(\phi, \chi)=c, \\
\Pi(\phi, \chi)=-\mu^{2} \kappa_{\phi} \phi^{2}-\kappa \chi^{2}-\frac{\beta}{4} \phi^{4}+\frac{3 s}{2} \frac{\chi^{2} \phi^{2}}{1-s \phi^{2}},
\end{gathered}
$$

where $c$ is an integration constant (a combination of the previous ones). It is important to notice that this is not the constant of motion that is the Hamiltonian of the full system:

$$
\chi_{0}^{2}=\frac{\phi_{0}^{2}\left(1-s \phi_{0}^{2}\right)\left(\beta \phi_{0}^{2}+4 \mu^{2} \kappa_{\phi}\right)}{2\left[s \phi_{0}^{2}(3+2 \kappa)-2 \kappa\right]} .
$$

In order for localized solutions to exist, it is clear that $\chi_{0}$ must be real. This leads to several conditions on the other parameters of the problem. First, the potential $\Pi$ has a singularity at $1-s \phi_{*}^{2}=0$ and limits the subsequent analysis to the regime $1-s \phi^{2}>0$, which, in physical terms, limits us to densities lower than the critical one corresponding to $\phi_{*}^{2}=1$. The laser equation (12) can be considered as a stationary Schrödinger equation for $\chi(x)$, and a localized solution requires a trapping potential, that is, $s=+1$, given the previous condition on the atom density. Once these conditions are satisfied, the existence of a localized solution to (12) further requires a negative eigenvalue, that is, $\kappa>0$. [Because the trapping potential for the laser field is due to the presence of $\phi(x)^{2}$, it must tend to zero as $x \rightarrow \pm \infty$.] The same must be true for the eigenvalue of the atom equation, that is, $\kappa_{\phi}>0$. Finally, in the parameter regime $s=+1, \beta>0$ (repulsive atomic collisions), $1-s \phi^{2}>0, \kappa_{\phi}, \kappa>0$, Eq. (17) implies that the existence of a real value for $\chi_{0}$ requires $1>\phi_{0}^{2}>\phi_{\min }^{2}=2 \kappa /(3+2 \kappa)$.

Therefore, what happens when the system has reached a stationary state with mutual localization can be understood in a more physical way. The laser plays the role of a trapping potential for the atoms as a consequence of the induced dipole-dipole interaction between them, which turns out to be attractive in the red-detuned case. At the same time, the atoms modify the refractive index experienced by the laser, with a consequent focusing effect on the electromagnetic radiation. Stationary localization requires an optimal balancing of the mutual effects: The atoms' focusing effect must be strong enough to counteract the natural diffractive spreading of the laser, and the laser trapping effect must be strong enough to counteract the natural tendency of the atoms to broaden away due to both kinetic energy and atomic repulsion.

\section{B. Low-density atomic beam}

In the regime of very low atom densities, the coupled equations admit analytical solutions that are interesting since they illustrate the fundamental solitonlike nature of possible mutually localized solutions. If we assume $\phi_{0}^{2} \ll 1$, Eqs. (11) and (12) reduce to

$$
\begin{gathered}
\phi^{\prime \prime}+\phi\left(-2 \mu^{2} \kappa_{\phi}-\beta \phi^{2}+s a^{2}+2 a^{2} \phi^{2}\right) \simeq 0, \\
a^{\prime \prime}+a\left(-2 \kappa+3 s \phi^{2}\right) \simeq 0,
\end{gathered}
$$

where $f^{\prime \prime}$ denotes the second derivative of a function $f$ with respect to $x$. Let $L_{a}$ and $L_{\phi}$ represent the characteristic length scales of the laser and atom in the solution. In order to have mutual localization it is necessary that $L_{a} \geqslant L_{\phi}$. In fact, in the opposite case $L_{a} \ll L_{\phi}$, the laser field would experience only a constant refractive index induced by the atoms; that is, the term $3 s \phi^{2}$ in Eq. (19) would be a constant, and this could not justify the localization of the stationary laser solution. On the other hand, even in the low-density limit, the atoms are subject to an induced dipole-dipole interaction which depends not only on the laser intensity distribution but on the atom density distribution as well [the last term of Eq. (18)]. This allows the existence of stationary solutions with a narrow atom and a broad laser distribution, and the absence of such a self-interaction term for the laser is the main reason why no solutions are expected to be found with $L_{a} \ll L_{\phi}$. Even though the discussion is simpler in the low-density limit, the same physical mechanism is at work in the full model, and we can anticipate here that, after a numerical investigation, we have not found any such solution, as will be shown in Fig. 3 and discussed in Sec. III C.

Thus both a single- and a multiple-scale mutually localized solution are in principle allowed in the low-density limit. For the single-scale solution, if we assume $a(x)=\alpha \phi(x)$ with $\alpha$ as a proportionality constant, the last term in the atom equation can be neglected, and the two equations transform into

$$
\begin{gathered}
\phi^{\prime \prime}+\phi\left(-2 \mu^{2} \kappa_{\phi}-\beta \phi^{2}+s \alpha^{2} \phi^{2}\right) \simeq 0, \\
\phi^{\prime \prime}+\phi\left(-2 \kappa+3 s \phi^{2}\right) \simeq 0 .
\end{gathered}
$$

The requirement that these equations must be identical gives the conditions $\mu^{2} \kappa_{\phi}=\kappa$ and $s \alpha^{2}-\beta=3 s$. The corresponding solution is the classical soliton solution

$$
\begin{gathered}
\phi(x)=2 \sqrt{\frac{s \kappa}{3}} \operatorname{sech}(\sqrt{2 \kappa} x), \\
a(x)=\sqrt{3+s \beta} \phi(x),
\end{gathered}
$$

which exists for $\beta>-3$.

In the case of multiscale solutions, the last term in the atom equation can no longer be dropped since $L_{a} \gg L_{\phi}$. It is precisely this term that allows the formation of localized atomic structures. Furthermore, this condition on the length scales of the two solutions allows one to replace $a(x)$ with 
its peak value $a_{0}=a(0)$ in the atom equation, since the laser amplitude varies very slowly with respect to the atom wave function. Thus, for the atoms we have again a hyperbolicsecant-shaped soliton solution

$$
\begin{aligned}
\phi(x)= & \sqrt{\frac{2 \mu^{2} \kappa_{\phi}-s a_{0}^{2}}{a_{0}^{2}-\beta / 2}} \text { sech } \\
& \times\left(\sqrt{\frac{\left|\beta / 2-a_{0}^{2}\right|}{\beta / 2-a_{0}^{2}}\left(s a_{0}^{2}-2 \mu^{2} \kappa_{\phi}\right) x}\right),
\end{aligned}
$$

while for the laser the same condition on the scale lengths implies that, over most of the domain of existence of the laser solution, the atom wave function will be negligibly small. Outside a central region of the order of $L_{\phi}$, the solution will be given by

$$
a(x)=a_{0} e^{ \pm \sqrt{2 \kappa} x} .
$$

By integrating the laser equation (19) once in the limit $L_{\phi} \rightarrow 0$ [which makes it possible to replace $a(x)$ with its peak value $a_{0}$ ] and using the solution found in (21), we obtain an expression for $\kappa$,

$$
\kappa=\frac{9}{2} \frac{2 \mu^{2} \kappa_{\phi}-s a_{0}^{2}}{\left(a_{0}^{2}-\beta / 2\right)^{2}} .
$$

The condition from Eq. (23) that $\kappa$ must be positive, combined with the condition that the square-root terms in Eq. (21) must be real, leads to the requirement $a_{0}^{2}>\beta / 2$ on the laser peak intensity in order for the stationary solution to exist, which limits the multiscale structure to the high-field regime. However, as can be seen, for instance, from the potential $\Pi(\phi, \chi)$, which has a singularity, these solutions will not necessarily be realizable since they may be unstable.

A direct variational approach, using trial functions and Ritz optimization, can be used to find approximate solutions in the multiscale case. This analysis is based on the Lagrangian corresponding to Eqs. (11) and (12), which is given by $L=\frac{1}{2}(d \phi / d x)^{2}+\frac{1}{2}(d \chi / d x)^{2}-\Pi$. Using trial functions of Gaussian form for simplicity, that is, $\phi=A \exp \left(-x^{2} / 2 a^{2}\right)$ and $\chi=B \exp \left(-x^{2} / 2 b^{2}\right)$, inserting these into the variational integral, and performing an integration over the independent variable $x$ from $-\infty$ to $+\infty$, one obtains an averaged Lagrangian $\langle L\rangle$ that depends on the parameters of the trial function $(A, B, a, b):\langle L\rangle=\langle L\rangle(A, B, a, b)$. Variation with respect to these parameters gives a system of four equations, which determines the parameters (for given $\kappa$ ). This approach has been used successfully to analyze a number of different problems in nonlinear optics (see, e.g., [10]). Unfortunately, the averaged expression for the full nonlinearity cannot be evaluated analytically, but if the analysis is restricted to the first-order expansion of the singular term, the integration can be performed and the subsequent variational equations can be expressed in explicit algebraic form and easily solved numerically. The analysis is straightforward (albeit semianalytical), but it does allow approximate solutions to be found for the multiscale case. An example of such a solution is given in Fig. 1 and compared to the numerically obtained full solution. The agreement is seen to be rather good except out in the wings of the distributions, where the Gaussian form of the

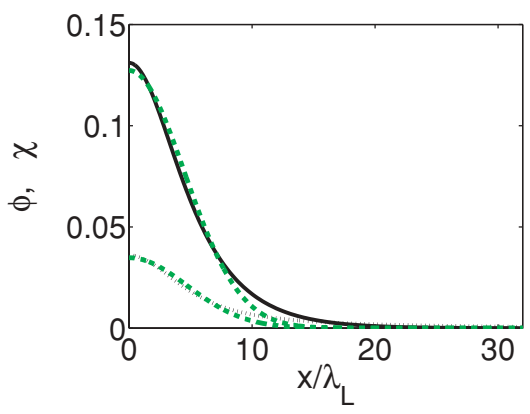

FIG. 1. (Color online) Comparison between results obtained using the numerical shooting method and the approximate variational approach for the atom wave function (dotted line) and the laser amplitude (solid line). The variational results are the two green dashed lines. The chosen parameters are $\kappa=10^{-3}, k_{\phi}=0.0905$, $\beta=38.4281$, and $\mu=1$. All quantities are dimensionless, the normalization is as in the text, and the coordinate $x$ is measured in units of the laser wavelength $\lambda_{L}$.

trial functions tends to decay more rapidly than the one found numerically [with the correct variation being exponential; $\mathrm{cf}$. Eq. (22)].

\section{General case}

Outside the low-density regime, the general solution to the coupled system of Eqs. (11) and (12) can be found only numerically, for instance, via the shooting method. This method is particularly suitable for the search for localized solutions. For fixed values of the eigenvalues $\kappa$ and $\kappa_{\phi}$, the method requires one to make a guess at the peak laser amplitude and atom density, solve the two coupled equations, which are now ordinary differential equations, and vary the guess until the solution found is a localized, single-hump solution for both atom and laser (see, for instance, [11]). Some examples of the localized solutions found for different values of $\kappa_{\phi}$ and $\kappa$ are shown in Fig. 2. In all these calculations it has been assumed that $\beta=38$, consistent, for instance, with a detuning of $100 \Gamma$ for ${ }^{87} \mathrm{Rb}$ atoms. The solutions become narrower and more peaked for increasing values of $\kappa$ and $\kappa_{\phi}$ as can be seen in Fig. 3, which shows the variation of the ratio of the widths of the laser and atom profiles for two different values of $\kappa_{\phi}$ and varying $\kappa$. However, all the solutions found numerically are such that the laser intensity distribution is never narrower than the atom density distribution, thus confirming the conclusion reached in Sec. III B in the analysis of the low-density case that solutions with $L_{a} \ll L_{\phi}$ are impossible.

Figure 2 also shows the change in the nature of the solution when we move from very low densities to higher ones. The atom wave functions tend not only to become more peaked but also more and more narrow as a result of the more important effect of the dipole-dipole nonlinearity. This hints as well at a possible change in the stability characteristics of the solutions, as will be discussed in the next section.

\section{STABILITY ANALYSIS}

Using the slowly varying envelope approximation, well known in nonlinear optics, we write the atom and laser wave 

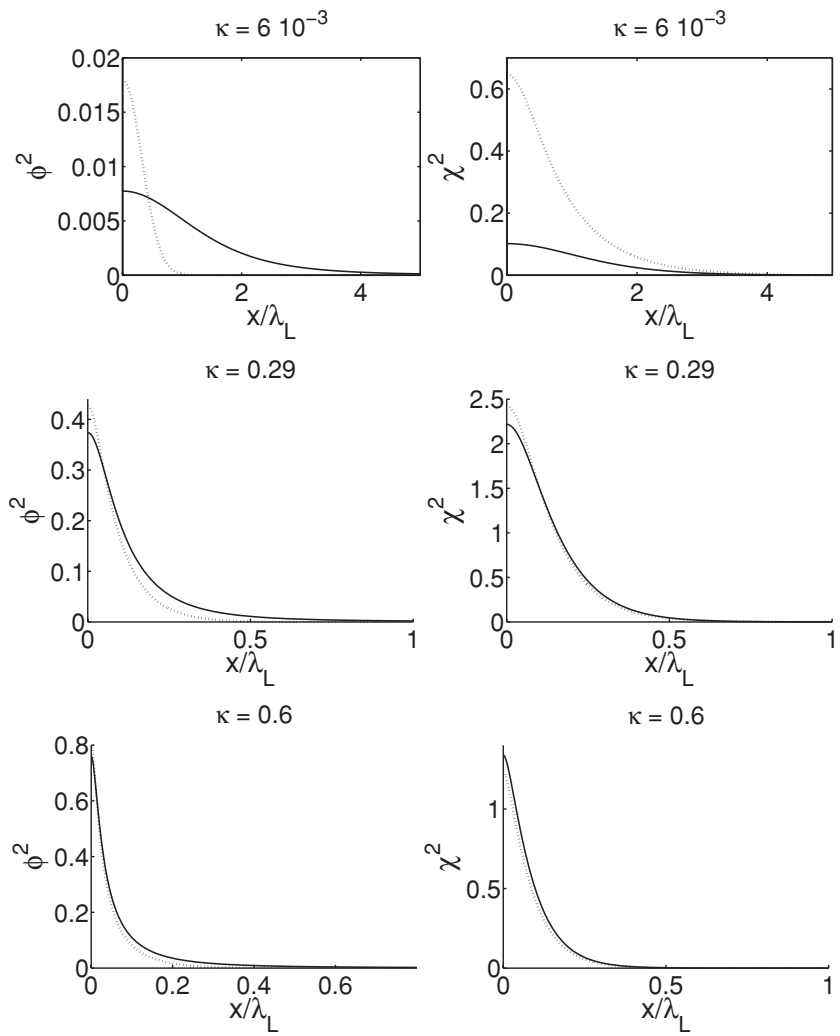

FIG. 2. Examples of stationary solutions calculated numerically. $\kappa$ is indicated in the plots; $\kappa_{\phi}=6 \times 10^{-4}$ (solid line) and $6 \times 10^{-1}$ (dotted line). All quantities are dimensionless, the normalization is as in the text, and the coordinate $x$ is measured in units of the laser wavelength $\lambda_{L}$. Here $\beta=38.4281$ and $\mu=1$.
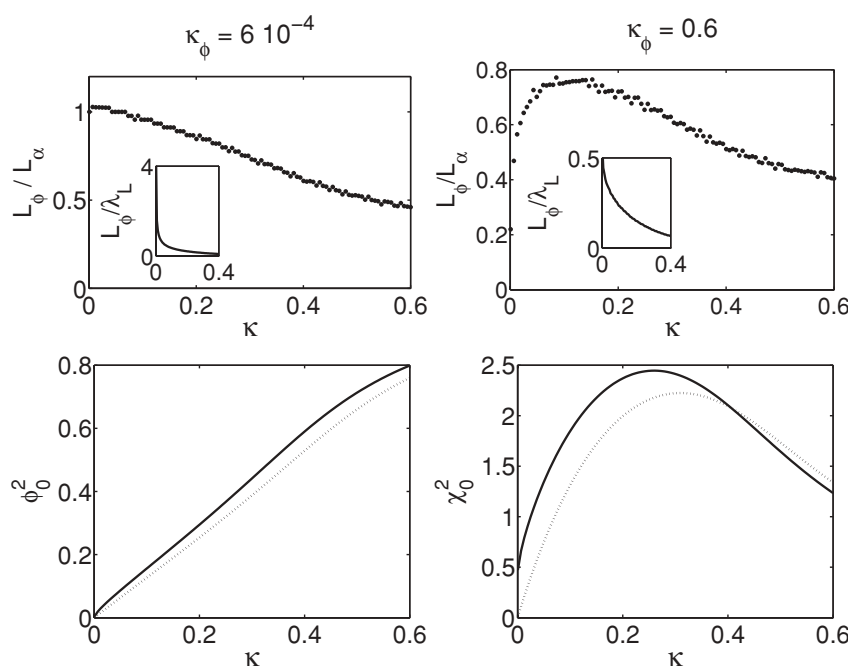

FIG. 3. Top row: Variation of the atom width $L_{\phi}$ with respect to the laser width $L_{a}$ versus $\kappa$ for two fixed values of $\kappa_{\phi}$. In the inset the actual atom width $L_{\phi}$ is shown in units of $\lambda_{L}$ versus $\kappa$ for the same values of $k_{\phi}$. These widths are calculated as the widths at which the density (or intensity) has been reduced to $1 / e$ of its peak value. Bottom row: Variation of the atom peak density $\phi_{0}$ and laser peak intensity $\chi^{2}$ for $\kappa_{\phi}=6 \times 10^{-4}$ (dotted line) and 0.6 (solid line) versus $\kappa$. All quantities are dimensionless, the normalization is as in the text, $\beta=38.4281$, and $\mu=1$. functions as

$$
\begin{aligned}
& \mathbf{E}(\mathbf{r})=a\left(\mathbf{r}_{\perp}, z\right) \exp (i k z) \mathbf{e}, \\
& \Phi(\mathbf{r})=\phi\left(\mathbf{r}_{\perp}, z\right) \exp \left(i k_{a} z\right),
\end{aligned}
$$

where the complex amplitudes $a\left(\mathbf{r}_{\perp}, z\right)$ and $\phi\left(\mathbf{r}_{\perp}, z\right)$ are assumed to be slowly varying in the direction of propagation and obey the following normalized coupled nonlinear parabolic equations:

$$
\begin{gathered}
i \mu \frac{\partial \psi}{\partial z}=-\frac{1}{2} \nabla_{\perp}^{2} \psi+\frac{1}{2} \beta|\psi|^{2} \psi-\frac{s}{2} \frac{|a|^{2}}{\left(1-s|\psi|^{2}\right)^{2}} \psi, \\
i \frac{\partial a}{\partial z}=-\frac{1}{2} \nabla_{\perp}^{2} a-\frac{3 s}{2} \frac{|\psi|^{2} a}{1-s|\psi|^{2}} .
\end{gathered}
$$

(The normalization is as before.) The system of Eqs. (26) and (27) corresponds to the Hamiltonian density

$$
\mathcal{H}=\frac{1}{2}\left|\nabla_{\perp} \psi\right|^{2}+\frac{1}{2}\left|\nabla_{\perp} a\right|^{2}+\frac{\beta}{4}|\psi|^{4}-\frac{s}{2} \frac{|a|^{2}|\psi|^{2}}{\left(1-s|\psi|^{2}\right)} .
$$

In addition to the Hamiltonian $H=\int_{-\infty}^{+\infty} \mathcal{H} d^{2} \mathbf{r}_{\perp}$, the system also admits more constants of motion such as the total momentum and the two Manley-Rowe integrals

$$
N=\int_{-\infty}^{+\infty}|\psi|^{2} d^{2} \mathbf{r}_{\perp}, \quad P=\int_{-\infty}^{+\infty}|a|^{2} d^{2} \mathbf{r}_{\perp} .
$$

The Hamiltonian is not generally limited from either below or above, and therefore specific information about the stability problem can be drawn computationally only, for example, by direct modeling of Eqs. (26) and (27). However, the results of a detailed analysis of this problem will be presented elsewhere. On the other hand, in the low-density limit, useful information can be obtained analytically by neglect of the denominator in the dipole-dipole term:

$$
\begin{aligned}
H \simeq & \int_{-\infty}^{+\infty}\left(\frac{1}{2}\left|\nabla_{\perp} \psi\right|^{2}+\frac{1}{2}\left|\nabla_{\perp} a\right|^{2}\right. \\
& \left.+\frac{\beta}{4}|\psi|^{4}-\frac{s}{2}|a|^{2}|\psi|^{2}\right) d^{2} \mathbf{r}_{\perp},
\end{aligned}
$$

where for the last term

$$
\int_{-\infty}^{+\infty}|a|^{2}|\psi|^{2} d^{2} \mathbf{r}_{\perp} \leqslant N P
$$

which means that for the case of $\beta>0$ the Hamiltonian is limited from below. The same conclusion can be drawn also for $\beta<0$ but for a one-dimensional geometry $\left(\mathbf{r}_{\perp}=x\right)$ only. For systems with more than one transverse dimension, as is well known in nonlinear optics (see, e.g., [12] and references therein), a collapsing regime may be reached. This can happen even in the case of one transverse dimension if the full denominator of the dipole-dipole term is taken into account. When the Hamiltonian is limited from below, we can try to 
find a class of solutions that minimize $H$, and a Lyapunov analysis in this case provides a clear indication that single-scale structures are stable. In fact, by application of the method of Lagrangian multipliers with

$$
\tilde{H}=H+\lambda_{\phi} N+\lambda_{\varepsilon} P,
$$

the complex variation of $\tilde{H}$ yields exactly the equations for single-scale solitons provided $\lambda_{\phi}=\mu^{2} \kappa_{\phi}$ and $\lambda_{\varepsilon}=\kappa$, that is, the single-scale soliton solutions minimize the functional $\tilde{H}$. Since this functional consists of integrals of motion (28) and (29) only, it can be treated as a Lyapunov functional. Thus, for all perturbations we have $\frac{d \tilde{H}}{d z}<0$, which proves the stability of the single-scaled soliton solutions against small perturbations.

Although the stability of the low-density, single-scale solutions has been demonstrated here, the presence of the singularity in the laser-induced nonlinear term makes the problem of the stability of the solutions in the fully nonlinear case an open question. A rigorous answer to the stability question would require a study of the eigenvalues of the perturbed linearized system. However, a good indication of the fate of these structures comes from numerical simulations of their evolution according to the propagation equations (26) and (27). It is found that for relatively low values of $\kappa$ and $\kappa_{\phi}$ the stationary structures are robustly stable, propagating effectively unchanged even if slightly perturbed initially. For higher values of $\kappa_{\phi}$ and still relatively low $\kappa$, the stationary structures during propagation evolve toward different stationary structures, still mutually localized but with lower peak atom density and laser intensity. On further increase of $\kappa$, the structures show a clear sign of instability, leading to yet another scenario in which a minimal increase in $N_{0}$ leads to collapse while a decrease leads to broadening and total loss of localization of the structures. Figure 4(a) shows a collapse threshold curve versus the two eigenvalues $\kappa$ and $\kappa_{\phi}$ while Fig. 4(b) shows the corresponding peak atom densities and laser intensities; Figs. 4(c) and 4(d) show the corresponding widths of these structures at the threshold. These last two plots are an indication that the stability region is actually broader than we found here. In fact, this threshold curve was found by numerical propagation of the stationary structures according to Eqs. (26) and (27), which are valid under the paraxial approximation. However, although the equations used to calculate the stationary solutions are exact, the evolution of the solutions corresponding to the collapse threshold of Fig. 4 must be described through the full equations with no paraxial approximation, since their widths are smaller than the laser wavelength. It is known from previous investigations in the field of nonlinear optics (see, for instance, [13]) that the extra terms together make the structures stable beyond the limits found under the paraxial approximation, a result of more effective diffraction effects for such narrow structures. Thus we can safely assume that we have an even broader stability region. This result indicates the existence of a regime of parameters in which the mutually localized structures propagate stably with unchanged form.

As mentioned previously, numerical simulations of the propagation equations starting from initial distributions that are not the calculated stationary ones show that it is possible
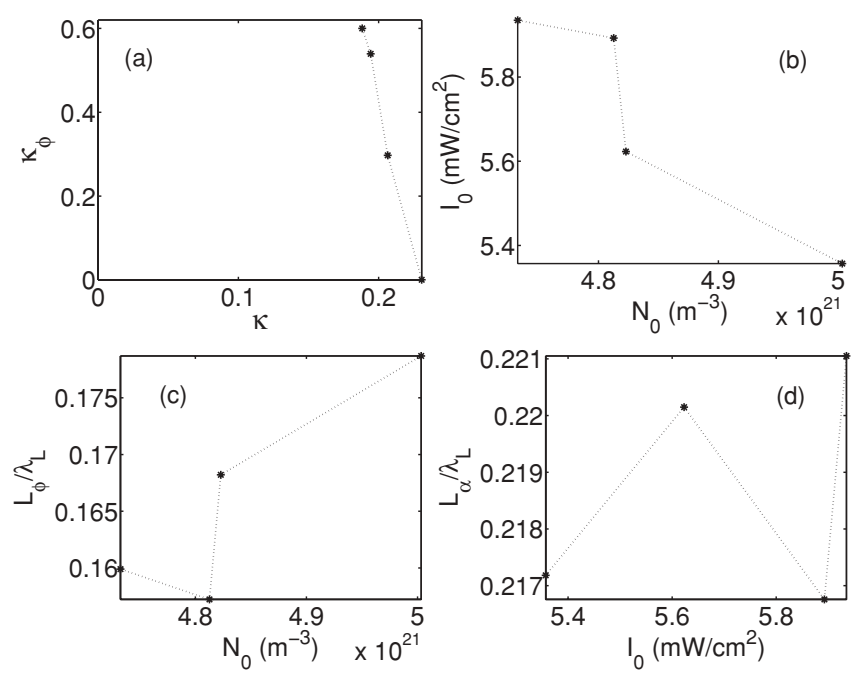

FIG. 4. Collapse threshold of the stationary solutions found numerically. (a) Structures corresponding to eigenvalues above the dotted line show a collapsing evolution. All quantities are dimensionless and the normalization is as in the text. (b) Peak atom density and laser intensity at the collapse threshold points corresponding to the eigenvalues shown in (a). Again, structures above this threshold line show a collapsing evolution. (c) and (d) Widths of atom and laser structures measured in laser wavelengths, corresponding to the points in (b). Here $\beta=38.4281$ and $\mu=1$.

to excite structures such as single- and two-scale solitons and also that there is a parameter range in which they are stable [4].

\section{CONCLUSIONS}

Based on a semiclassical approach we have derived a set of self-consistent equations describing the interaction of a BEC with laser fields. These equations were then applied to the problem of mutual guiding of laser and BEC atomic beams. Within this framework, stationary solutions of the nonlinear system describing possible configurations of laser and BEC atom beam guiding were found. Numerical simulations of the coupled propagation seem to indicate the possibility of reaching stationary and mutually localized states, and we have presented here both analytical (in the low-density limit) and numerical stationary solutions. A study of the stability of these structures shows that there is a parameter region in which they are stable, and the presence of stability regions should make this effect observable experimentally. A possible experimental setup to reflect these results would require a two-dimensional condensate initially localized in what here was defined as the transverse dimension and weakly localized along the propagation direction of the radiation (with one possibility being its use as a gravito-optical trapping system). With the interaction of the incident laser beam, a transient is to be expected along the propagation direction of the radiation, with the two systems finally settling down to their own self-localized structures. For instance, for the cases presented in Fig. 4, considering ${ }^{87} \mathrm{Rb}$ atoms and a red detuning of about $100 \Gamma \sim 3.81 \mathrm{GHz}$ from the $5^{2} S_{1 / 2}-5^{2} P_{3 / 2}$ atomic resonance, we may expect structures with peak atom densities of the order of $5 \times 10^{21} \mathrm{~m}^{-3}$. In these cases the atomic beam has a longitudinal velocity of the order of $0.6-0.9 \mathrm{~cm} / \mathrm{s}$ and the 
number of atoms per unit longitudinal length is of the order of $10^{8} \mathrm{~m}^{-1}$. Of course, all the parameters, including the required laser intensity, naturally depend on the atoms chosen and on the detuning.
[1] J. J. Javanainen, Phys. Rev. Lett. 72, 2375 (1994).

[2] S. Giovanazzi, D. O’Dell, and G. Kurizki, Phys. Rev. Lett. 88, 130402 (2002); D. O’Dell, S. Giovanazzi, G. Kurizki, and V. M. Akulin, ibid. 84, 5687 (2000).

[3] W. Zhang and D. F. Walls, Quantum Opt. 5, 9 (1993); M. Lewenstein, Li You, J. Cooper, and K. Burnett, Phys. Rev. A 50, 2207 (1994); M. Saffman, Phys. Rev. Lett. 81, 65 (1998); K. V. Krutitsky, F. Burgbacher, and J. Audretsch, Phys. Rev. A 59, 1517 (1999).

[4] F. Cattani, A. Kim, D. Anderson, and M. Lisak, J. Phys. B: At. Mol. Opt. Phys. 43, 085301 (2010).

[5] F. Cattani, D. Anderson, A. Kim, and M. Lisak, JETP Lett. 81, 561 (2005).

[6] H. L. F. v. Helmholtz, Wied. Ann. 13, 385 (1881).

[7] L. P. Pitaevskii, Sov. Phys. JETP-USSR 12, 1008 (1961).
[8] M. Born and E. Wolf, Principles of Optics: Electromagnetic Theory of Propagation, Interference and Diffraction of Light (Cambridge University Press, Cambridge, 1999).

[9] J. D. Jackson, Classical Electrodynamics (John Wiley and Sons, New York, 1998).

[10] D. Anderson, Phys. Rev. A 27, 3135 (1983).

[11] W. H. Press, S. A. Teukolsky, W. T. Vetterling, and B. P. Flannery, Numerical Recipes (Cambridge University Press, New York, 2007), Chap. 18.1.

[12] J. J. Rasmussen and K. Rypdal, Phys. Scr. 33, 481 (1986); C. Sulem and P. L. Sulem, Nonlinear Schrödinger Equation: Self-Focusing Instability and Wave Collapse (Springer, New York, 1999).

[13] N. N. Rozanov, N. V. Vysotina, and A. G. Vladimirov, JETP 91, 1130 (2000). 